\title{
Absorption Enhancement in Solar Cells With Periodic Interface Textures of Asymmetric Shape
}

\author{
Franz-Josef Haug, Karin Söderström, and Christophe Ballif
}

\begin{abstract}
We investigate light scattering interface textures for solar cells with the intention to obtain a more fundamental understanding of the absorption enhancement. Therefore, we study simplified structures with the periodic interface texture of sinusoidal and blazed shape, and we employ a theoretical model with moderate complexity on the basis of the Rayleigh method. We test our model by comparing diffraction effects with theoretical predictions for the two possible polarization directions. The intensity of first-order diffraction is virtually insensitive to the asymmetry of the grating for both s- and p-polarizations. However, in the latter case, the absorption signature of the surface plasmon resonances does show a mild asymmetry. Subsequently, we extend the model to multilayers such as a full solar cell stack, which allows us to relate the measured absorption enhancement to the interface texture.
\end{abstract}

Index Terms-Absorption, amorphous silicon, diffraction.

\section{INTRODUCTION}

A BSORPTION enhancement is desirable in a variety of applications ranging from sensors to optoelectronic elements and solar cells. In the past, many ideas have been put forth for silicon-based devices, because close to their band edges the absorption of all types of silicon is much weaker than that of semiconductors with a direct bandgap. Expressed in optical terminology, absorption enhancement is the incoupling of radiation into an active film. The concept is also an interesting option to reduce the amount of costly semiconductor materials in other technologies. Moreover, measures for enhanced incoupling apply equally for enhanced outcoupling of radiation, making the underlying ideas also applicable to increase the radiative efficiency of light-emitting diodes.

Experimentally, the most successful concept of absorption enhancement in solar cells is based on interface textures. Random or quasi-random structures are widespread because of their ease of fabrication, low manufacturing cost, and wideband operation [1]; periodic structures are expected to provide higher absorption enhancement; however, they might be restricted to narrower spectral regions or a narrower range of incident angles [2]. For bulk material, the textures are usually large and easily

Manuscript received July 2, 2015; revised August 18, 2015; accepted September 3, 2015. Date of publication October 1, 2015; date of current version October 19,2015 . This work was supported by the Swiss National Science Foundation (No. 200020_137700/1) and by the European Union within the FP7 projects FastTrack (No. 283501) and Cheetah (No. 609788).

F.-J. Haug and C. Ballif are with the Photovoltaics and Thin Film Electronics Laboratory, Institute of Microengineering, Polytechnique Fédérale de Lausanne, Neuchâtel CH-2000, Switzerland (e-mail: franz-josef.haug@epfl.ch; christophe.ballif@epfl.ch).

K. Söderström is now with the Centre Suisse d'Electronique et Microtechnique, PV-Centre, Neuchâtel CH-2000, Switzerland (e-mail: karin.soderstrom@ csem.ch).

Color versions of one or more of the figures in this paper are available online at http://ieeexplore.ieee.org.

Digital Object Identifier 10.1109/JPHOTOV.2015.2478024 described by geometric ray optics and ray tracing [2], [3]. For thin absorbing films, the textures should be scaled accordingly [4]. Here, one enters the more complex domain of wave optics and several state-of-the-art tools on the basis of finite-difference time domain [5]-[8], and the finite-element method [9], [10] were successfully used to handle typical cell configurations with random interface texture. However, the results are not always instructive or able to point out the underlying mechanisms of the absorption enhancement.

In this contribution, we study the mechanism of absorption enhancement in systems with textured interfaces. Therefore, we use a simplified device configuration with periodic interfaces instead of random ones, and we interpret our enhanced absorption as resonant diffraction into internal waveguide modes. Combining sinusoidal and mildly blazed interfaces with a Rayleigh method of moderate mathematical complexity, we are able to relate the coupling strength quantitatively to parameters like period, depth, and asymmetry of the grating.

\section{EXPERIMENTAL DETAILS}

The experimental results were carried out on line gratings that were obtained by replicating the commercial masters of blazed and sinusoidal shape (GR and GH series, 1800 lines per millimeter, Thorlabs) into an ultra-violet-curable resin (Ormocomp, MicroResist). The gratings were covered by $100-n m-$ thick silver films deposited by sputtering (Univex 450 B, Leybold). The reflection was measured from 320 to $1200 \mathrm{~nm}$ in a spectrophotometer (Lambda 900, Perkin Elmer) with polarized beam (ProFlux PPL05C, Moxtek). Diffuse and total reflections are measured with an integrating sphere under an incident angle of $8^{\circ}$ with respect to the surface normal. Angles closer to perpendicular incidence become accessible by rotating the polarizer and the sample simultaneously around the axis of the incident beam. The error introduced by the increasingly conical mount is minor. Angle-resolved diffraction efficiency was recorded with a home-built goniometer using laser excitation at $543 \mathrm{~nm}$.

Full solar cells were made on the Ag-covered gratings by adding a 60 -nm-thick $\mathrm{ZnO}$ buffer layer by sputtering. Next, a stack of n-doped, intrinsic, and p-doped amorphous silicon was deposited by plasma-enhanced chemical vapor deposition. The approximate thicknesses of the silicon films are 20, 220, and $10 \mathrm{~nm}$, respectively. Finally, a front electrode of sputtered ITO was deposited and structured to the desired cell size of $5 \times$ $5 \mathrm{~mm}^{2}$ by lift off. The ITO film is $65 \mathrm{~nm}$ thick and doubles as an antireflective layer.

Measurement of the surface topography with atomic force microscopy showed periods of about $550 \mathrm{~nm}$ and peak-to-valley 


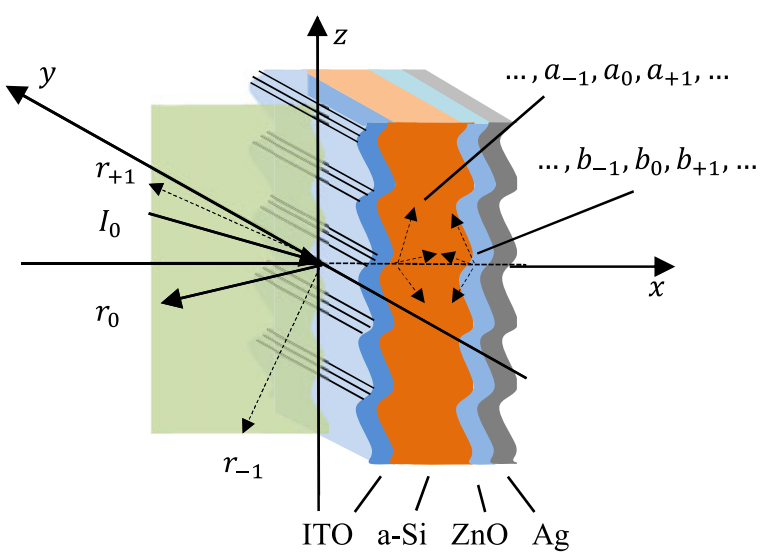

Fig. 1. Schematic presentation of the diffraction geometry and the expansion into forward and backwards propagating modes within the films.

depths of approximately $140 \mathrm{~nm}$; deviations from the master structure may be related to shrinking of the resist or residual stress after the deposition of films at high temperature.

\section{MODEL}

Diffraction from a periodic surface corrugation can be described with the Rayleigh method [11], which expands the electromagnetic fields in the media adjacent to the interface into diffraction modes and assumes that the expansion remains valid within the roughness zone of the corrugation up to the interface between the media. This latter assumption has been debated in the past, especially its applicability to deep corrugations [12], [13]. Experimental work [14] and numerical evidence [15] suggest the validity for the range of depths used in this contribution.

We use the geometry shown in Fig. 1, where the mean levels of the textured interfaces are parallel to the $y z$-plane, and the grating lines are oriented along the $y$-axis [16]. The period is denoted by $P$.

For a plane wave incident in a medium with $\varepsilon_{i}=1$, the wavelength $\lambda_{0}$ defines the wave vector modulus $k_{0}=2 \pi / \lambda_{0}$. The angle of incidence $\theta$ is measured with respect to the surface normal and yields wave vector components in $z$-direction as $k_{i}=$ $-k_{0} \sin \theta$. The $x$-component is defined by $\gamma_{i}=\left(k_{0}^{2}-k_{i}^{2}\right)^{1 / 2}=$ $k_{0} \cos \theta$. For the specularly reflected beam, we obtain $k_{r}=$ $+k_{0} \sin \theta$ and $\gamma_{r}=\gamma_{i}$ for $z$ - and $x$-components, respectively. Electric field vectors oriented parallel to the $y$-direction correspond to s-polarization in Fresnel notation (TE in waveguide notation). Likewise, magnetic fields parallel to the $y$-axis denote p-polarization (corresponding to TM). The presence of the periodic surface corrugation is taken into account by reciprocal grating vector $G=2 \pi / P$. Thus, the $z$-component of the diffracted wave vector of $p$ th order is defined via $k_{p}=k_{0} \sin \theta+p G$; this definition holds for all films because the inplane component is conserved across all interfaces. The $x$-components must be determined individually for each medium or film $f$; for a permittivity $\varepsilon_{f}$, they are defined via $\gamma_{f, p}=\left(\varepsilon_{f} k_{0}^{2}-k_{p}^{2}\right)^{1 / 2}$. The reflected field in the incident medium contains thus either purely real or purely imaginary contributions. The former represent propagating diffraction orders, and the latter are evanescent waves which do not propagate and do not carry energy. In media or films with complex permittivity $\varepsilon_{f}=\varepsilon_{f}^{\prime}+i \varepsilon_{f}^{\prime \prime}$, the definition yields waves with exponential attenuation along the $x$-direction.

Different from the iterative treatment used by L. Rayleigh [11], insertion of the expanded electromagnetic fields into the boundary conditions yields an infinite set of linear algebraic equations that is mathematically rigorous [14], [17], [18]. An approximate solution is obtained by truncating to desired order $m$, resulting in $2 \cdot(2 m+1)$ equations per interface; the coefficients contain only the wave vector components and instances of an integral transformation of the function $x=g(z)$ that describes the interface

$$
X_{p}\left(\gamma_{f, p}\right)=\frac{1}{P} \int_{-P / 2}^{P / 2} e^{-i p G z} e^{i \gamma_{f, p} g(z)} d z .
$$

For a sinusoidal interface given by $g(z)=\sigma \cdot \sin G z$, (1) is a representation of $J_{p}\left(\gamma_{f, p} \sigma\right)$, which is the Bessel function of order $p$. The resulting system of equations is then particularly compact (c.f. [19], (2.8) to (2.11) for the case of a film on a sinusoidally textured substrate), and it is possible to evaluate the absorption in the individual layers of a stack without reverting to numerical integration [16].

Other than sinusoidal shapes, it is also possible to evaluate (1) for piecewise linear functions like binary gratings, sawtooths, or triangular shapes [14], but we are not aware of any studies that examined the effect of discontinuous or nondifferentiable functions on the accuracy of the approach.

For sinusoidal shapes with a mild second harmonic $g_{h}(z)=$ $\sigma_{1} \cdot \sin G z+\sigma_{2} \cdot \sin (2 G z+\phi), \sigma_{1}>\sigma_{2}$, we may expand the second exponential of (1) and obtain

$$
\begin{aligned}
X_{p}(\gamma)= & J_{p}\left(\gamma \sigma_{1}\right) \\
& +2 \gamma \sigma_{2} p \cdot\left(\left.\frac{\partial}{\partial t} \frac{J_{p}(t)}{t}\right|_{t=\gamma \sigma_{1}}\right), \quad \phi=0 \\
X_{p}(\gamma)= & \left(1+i \gamma \sigma_{2}\right) \cdot J_{p}\left(\gamma \sigma_{1}\right) \\
& +2 i \gamma \sigma_{2} \cdot\left(\left.\frac{\partial^{2}}{\partial t^{2}} J_{p}(t)\right|_{t=\gamma \sigma_{1}}\right), \quad \phi=\frac{\pi}{2} .
\end{aligned}
$$

Here, $\gamma$ is meant to represent $\gamma_{f, p}$ for given film $f$ and mode order $p$, and (2) and (3) represent the lowest order approximations of blazed and binary gratings, respectively. In (2), a positive amplitude $\sigma_{2}$ represents a backward (here: downwards) directed blaze as shown in Fig. 1. In (3), positive $\sigma_{2}$ and $\phi=+\pi / 2$ corresponds to an interface with broad peaks and pinched valleys, as shown by the dashed line in Fig. 2. For arbitrary values of $\phi$, (1) yields a weighted superposition of (2) and (3).

\section{RESUlTS AND DisCUSSION}

Fig. 2 shows a scanning electron cross-sectional micrograph of the blazed grating used here and in an earlier contribution [20]. Overlaid are measured AFM profiles obtained by averaging 512 lines of an image scanned perpendicular to the grating. The images were taken from a region between the cells where silicon was removed by dry etching, i.e., on the surface of the $\mathrm{ZnO} / \mathrm{Ag}$ back reflector, and on the surface of a cell, i.e., on the 


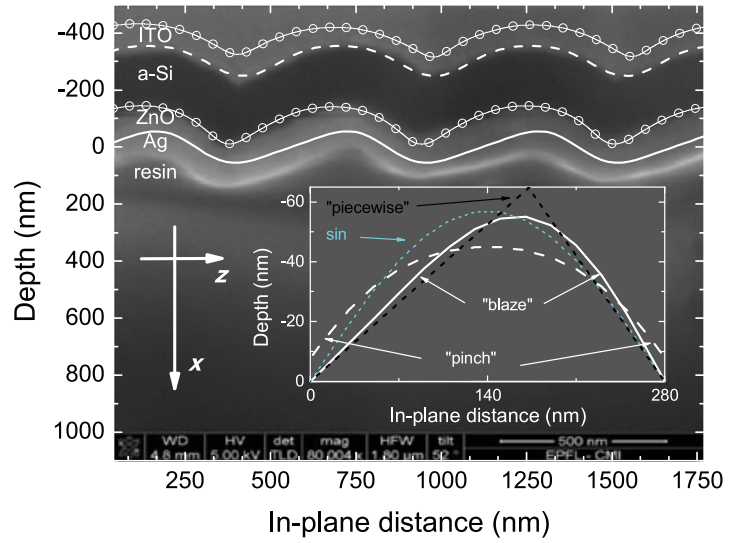

Fig. 2. Cross section micrograph of the multilayer structure. Overlaid symbols denote measured AFM data on ITO and on the ZnO covered Ag reflector. Full and dashed lines denote a sinusoidal of amplitude $\sigma_{1}=53 \mathrm{~nm}$ with second harmonic component of $\sigma_{2}=8 \mathrm{~nm}$, using $\phi=0$ and $\phi=\pi / 2$, respectively. (Inset) Two characteristics over a half period, including the pure sinusoidal and the piecewise linear approximation.
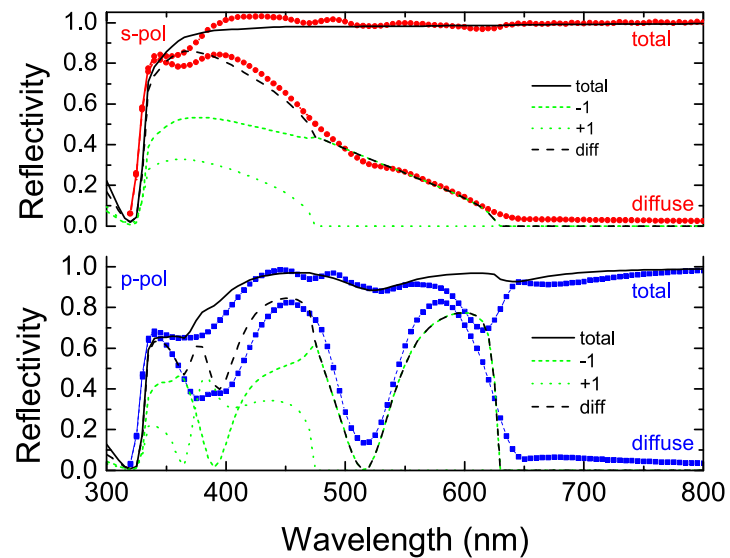

Fig. 3. Total and diffuse reflection of a sinusoidal grating with period of $550 \mathrm{~nm}$ for incidence of $8^{\circ}$. Upper and lower panels correspond to s- and ppolarization, respectively. Symbols represent measurements and lines denote modeled data that are additionally resolved for diffraction of -1 st and +1 st order.

ITO layer. Compared with the replication of the blazed grating, these surfaces are already considerably rounded by nonconformal growth. Owing to the small contrast between $\mathrm{ZnO}$ and $\mathrm{Ag}$, the surface of the silver covered grating is difficult to discern in Fig. 2. For the description of reflection experiments in the Sections B and C, we used either an idealized blaze represented by a piecewise linear function with amplitude of $65 \mathrm{~nm}$ and peak positions at $\pm 175 \mathrm{~nm}$ or an anharmonic sinusoidal with amplitudes of $\sigma_{1}=53 \mathrm{~nm}$ and $\sigma_{2}=8 \mathrm{~nm}$, using zero phase shift. For the cell results discussed in Section D, the broadened peak and the pinched valleys of the upper interfaces are described by the same amplitudes but using a phase shift of $\phi=+\pi / 2$.

\section{A. Reflection of Ag-Covered Sinusoidal Gratings}

Before discussing asymmetric gratings, we present results on symmetric gratings with sinusoidal shape $\left(\sigma_{2}=0\right)$. Fig. 3 shows total and diffuse reflection for a period of $550 \mathrm{~nm}$, a depth of $70 \mathrm{~nm}$, and an angle of incidence of $8^{\circ}$.

Reading from right to left, the reflection of s-polarized light is specular up to the onset of diffraction into -1 st order at $630 \mathrm{~nm}$. Toward shorter wavelengths, the diffracted intensity gradually increases until the onset +1 st order at $470 \mathrm{~nm}$. Bulk absorption in silver sets in below $340 \mathrm{~nm}$, the onset of $-2 \mathrm{nd}$ order at $315 \mathrm{~nm}$ is not measured. Overlaid are modeled characteristics using tabulated data for silver [21]. Typically, the sinusoidal grating yields numerically stable results for truncation to $m=2$ or $m=3$, i.e., systems of 10 or 14 equations, respectively [22]. However, we used fourth order throughout this contribution, including the modeling results of the full solar cell. The modeled spectra agree well with the measured data although the total reflection exceeds the theoretical expectation by about 3\% to $4 \%$ for wavelengths below $450 \mathrm{~nm}$. We attribute the inaccuracy to insufficient randomization of the diffracted beams with respect to the diffuse scatterer that we use for the normalization of the integrating sphere.

For p-polarization, -1 st and +1 st order have much stronger intensity directly at their onset, but at $520 \mathrm{~nm}$, there is a condition of almost completely specular reflection. The modeled results reproduce this feature. We also find deficiencies in the total reflection at 670,610 , and 520 , as well as below $400 \mathrm{~nm}$. The shallow one at $670 \mathrm{~nm}$ is also predicted theoretically, although at shorter wavelength of $640 \mathrm{~nm}$. The second one is not reproduced by the theory, but we can attribute it to an artifact of the measurement because directly after onset of the diffracted beams, they propagate under a grazing angle over the surface, and therefore, they are not efficiently captured into the integrating sphere. The absorption signature at $520 \mathrm{~nm}$ is well reproduced in position and extent, the agreement for the broad signature below $400 \mathrm{~nm}$ is acceptable, considering that this is also a region of limited accuracy for s-polarization.

The absorption signatures at 670 and $520 \mathrm{~nm}$ are related to the excitation of surface plasmon polaritons (SPPs). This is more easily seen in a dispersion diagram, i.e., by mapping the reflection data with respect to the energy of the incoming photons and their in-plane wave vector component $k_{\|}=k_{0} \sin \theta$.

For a moderate surface texture like the one used here, the dispersion relation of the SPP on a flat air/Ag interface may be used for illustration (c.f., [23, eq. (2.5)]):

$$
k_{\mathrm{SPP}}(\omega)=\frac{\omega}{c} \sqrt{\frac{\varepsilon_{\mathrm{Ag}}}{\varepsilon_{\mathrm{Ag}}+1}} .
$$

Instead of the usual notation for dispersion relations in the form of $\omega(k)$, we use $k(\omega)$ because the relative permittivity $\varepsilon_{\mathrm{Ag}}$ varies with photon energy. For silver, a good approximation is obtained by inserting only the real part $\Re\left(\varepsilon_{\mathrm{Ag}}\right)=\Re\left(\varepsilon^{\prime}+i \varepsilon^{\prime \prime}\right)$. Fig. 4 shows the dispersion relation of (4) in extended form and after reducing into the first Brillouin zone (BZ). Reflection data for angles of incidence between $0^{\circ}$ and $8^{\circ}$ is superimposed, representing the absorption $A=1-R$ by the symbol size.

The angular variation of the absorption dips between $670 \mathrm{~nm}$ $(1.85 \mathrm{eV})$ and $520 \mathrm{~nm}(2.4 \mathrm{eV})$ in Fig. 4 agrees well with the SPP characteristic after shifting by one reciprocal lattice vector from the second BZ into the central one. Likewise, the dips below 


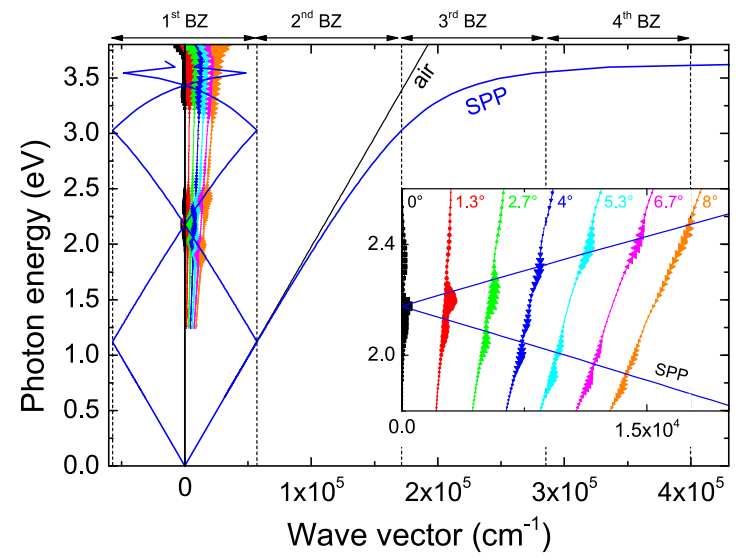

Fig. 4. Dispersion relation of the SPP resonance at the air/Ag interface using data of [21]. Dashed lines illustrate Brillouin zones for a period of $550 \mathrm{~nm}$, where the inset shows a magnification of the central zone. The symbol size scales with the measured absorption $(A=1-R)$.
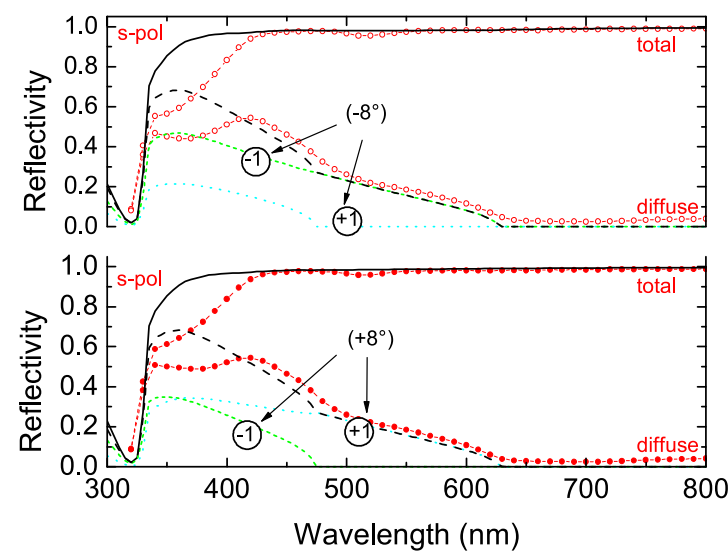

Fig. 5. Total and diffuse reflection of s-polarized light. The upper and lower panels show incident angles of $-8^{\circ}$ and $+8^{\circ}$, respectively. Lines illustrate theoretical characteristics using the piecewise approximation of Fig. 2, the angle of incidence is denoted by the number in parenthesis, and numbers inside circles denote mode orders.

$400 \mathrm{~nm}$ (above $3.1 \mathrm{eV}$ ) are attributed to shifts from the third BZs. Contributions of a higher order are difficult to distinguish from the bulk absorption in silver which sets in beyond $3.7 \mathrm{eV}$.

\section{B. S-Polarized Reflection From the Blazed Grating}

For all measurements of the blazed grating, we assume a mounting as illustrated in Figs. 1 and 2, where the downward (or left) face of the blaze is the longer one. The resulting reflection of s-polarized light for positive and negative angles of incidence is shown in Fig. 5. The spectral variation is very similar to the one of the sinusoidal grating in Fig. 3, but the intensity of the diffracted modes is smaller because of a slightly shallower corrugation. The results for incidence from negative or positive angles are virtually identical above $400 \mathrm{~nm}$. Some differences appear below this wavelength, but we saw in the previous section that the accuracy of our measurement is limited in this region.

In Fig. 5, the measured data are superimposed with theoretical characteristics, using a piecewise linear function for the de-

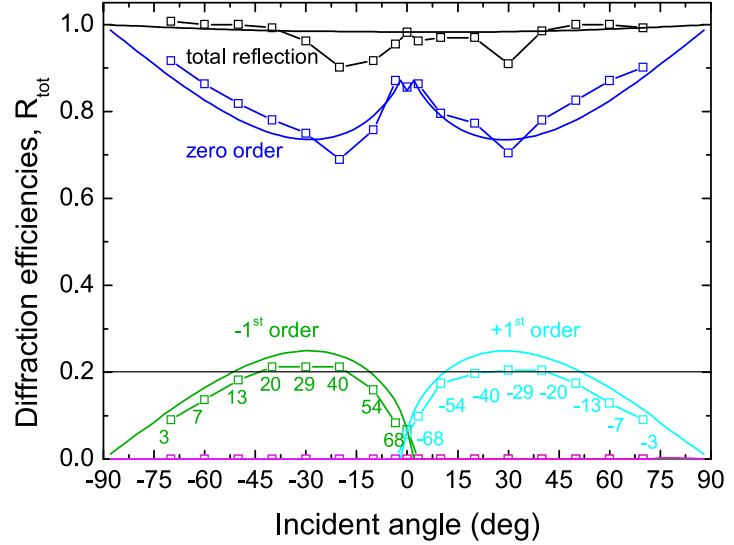

Fig. 6. Angular variation of the diffracted light intensity using a wavelength of $543 \mathrm{~nm}$. Symbols and lines denote measured and theoretical data, respectively. Numbers next to the symbols denote the diffracted angles of the first-order beams.
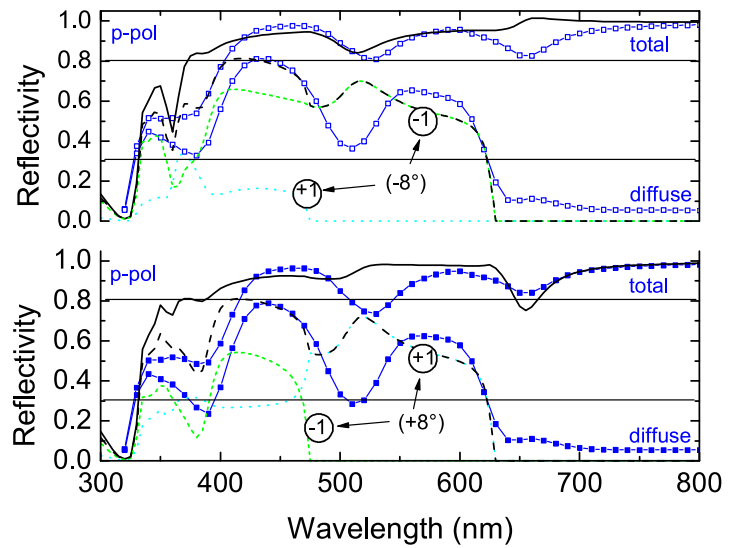

Fig. 7. Total and diffuse reflection of p-polarized light. The upper and lower panels show incident angles of $-8^{\circ}$ and $+8^{\circ}$, respectively and results of the piecewise approximation are denoted by lines.

scription of the interface. The modeled characteristics suggest that the purpose of the blaze, i.e., preference for one of the diffracted orders, becomes only noticeable in the spectral region where both orders propagate (here below $470 \mathrm{~nm}$ ). Modeling results on the basis of (2) are indistinguishable from the ones with the piecewise definition.

The angular variation for an incident wavelength of $543 \mathrm{~nm}$ is shown in Fig. 6. In addition, the difference between negative and positive angles of incidence is marginal and are only barely visible around the line drawn at 0.2 .

\section{P-Polarized Reflection From the Blazed Grating}

Fig. 7 shows reflection of p-polarized light from the blazed grating. In this case, the diffracted intensities are again very similar, but the horizontal lines at 0.8 and at 0.3 reveal noticeable differences in the SPP absorption signatures and in the intensity of the diffracted beams, especially around $500 \mathrm{~nm}$. The superimposed modeling results for the piecewise approximation are in poor agreement with the measured data, notably around the absorption signatures in the total reflection and in the diffracted 



Fig. 8. Total and diffuse reflection of p-polarized light like Fig. 7 but superimposed with modeled data using a sinusoidal with $\sigma_{1}=53 \mathrm{~nm}$ and a second harmonic with $\sigma_{2}=8 \mathrm{~nm}$.

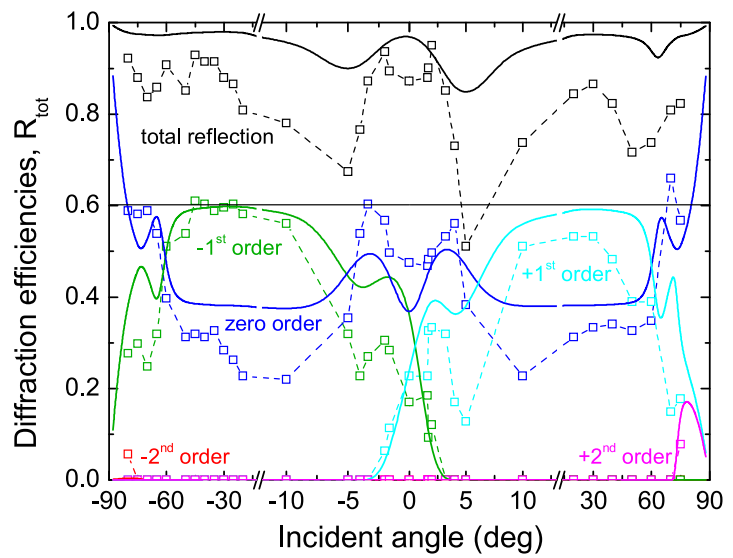

Fig 9. Total and diffracted light intensity of p-polarized light with respect to the incident angle using a wavelength of $543 \mathrm{~nm}$ (symbols). Modeled data (lines) uses $\sigma_{1}=53 \mathrm{~nm}$ and $\sigma_{2}=8 \mathrm{~nm}$, and the central region is expanded for clarity.

intensity around $500 \mathrm{~nm}$, where a peak is predicted instead of a dip.

Compared with the results with the piecewise linear function, Fig. 8 shows that modeling with the continuous and differentiable function underlying to (2) provides a significant improvement. Except for the problematic region below $400 \mathrm{~nm}$, the measured data of total and diffuse reflection are reproduced with acceptable accuracy. The SPP absorption dips are missed by ca. $8 \%$ in the total reflection, but the dip in diffracted intensity at $500 \mathrm{~nm}$ is reproduced very accurately, as illustrated by the horizontal line at 0.3 . Likewise, the line at 0.8 illustrates that the asymmetry of the absorption signatures for negative and positive angles of incidence at $500 \mathrm{~nm}$ is described correctly.

Finally, we present in Fig. 9 the angular variation of specular and diffracted intensities under p-polarization, again for a wavelength of $543 \mathrm{~nm}$. Similar to Fig. 6, we note that the measured total reflection at shallow angles is considerably below the modeled data. We attribute this shortcoming to our experimental setup because here, diffraction into grazing beams is collected poorly. Nevertheless, the measured intensity maxima of the zero

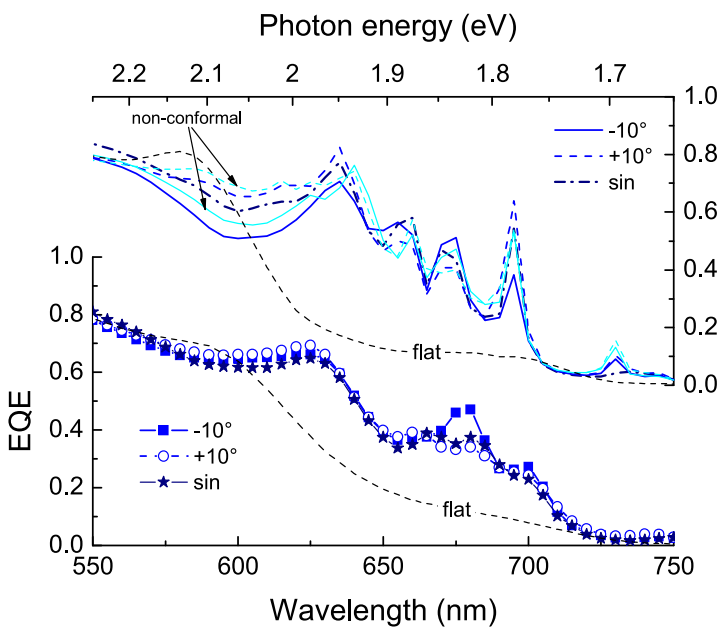

Fig. 10. Measured EQE (lower panel, left scale) and calculated absorption in the intrinsic layer (upper panel, right scale) of solar cells for p-polarized light. Open and closed symbols refer to incidence under $-10^{\circ}$ and $+10^{\circ}$ on the blazed structure, respectively, and stars denote an equivalent device on a symmetric grating with sinusoidal shape. Full, dashed, and dash-dotted lines represent the corresponding modelling results. Measured and modelled data for a flat reference are added in the lower and upper panels, respectively.

order beam at $\pm 4^{\circ}$ and of the first order beams at $\pm 3^{\circ}$, as well as the maxima at $\pm 75^{\circ}$, comply with modeled results.

\section{Solar Cell on the Blazed Grating}

The modeling routine is easily extended from a single interface to multilayers such as the full solar cell structure shown in Fig. 2. Experimentally, the absorption within the cell is characterized by the external quantum efficiency (EQE), which is the spectrally resolved probability of extracting an electron-hole pair from an incident photon. In the model, the EQE is approximated by integrating the local absorption over the region of the intrinsic layer only, i.e., excluding the absorption that takes place in the p-doped layer toward the front and in the n-doped layer at the back. Devices with sinusoidal interfaces have been discussed elsewhere [16]; here, we focus on interfaces with blazed shape. For the case of p-polarization, full and open symbols in Fig. 10 illustrate that $+10^{\circ}$ yields better response between 575 and $625 \mathrm{~nm}$, whereas the resonance at $675 \mathrm{~nm}$ is stronger for $-10^{\circ}$. For the modeling, we used either fully conformal interfaces according to (2) or a nonconformal combination of (3) for the front interfaces (air/ITO and ITO/a-Si) and of (2) for the back interfaces (a-Si/ZnO and $\mathrm{ZnO} / \mathrm{Ag}$ ).

The measured differences in the resonances are reproduced by the modeling results, although they are somewhat exaggerated. Overall, the model with nonconformal interfaces appears to yield a more appropriate description; notably, it avoids the severe dip predicted for incidence under $-10^{\circ}$ at $600 \mathrm{~nm}$. Similar features, including inconsistencies, are observed for the case of s-polarization (not shown).

Fig. 10 includes results of a cell on a fully symmetric grating with sinusoidal interfaces. Experimentally, this device performs worse than the blazed cell under either incident angle, but we observe that this is not consistently reproduced by the model; 


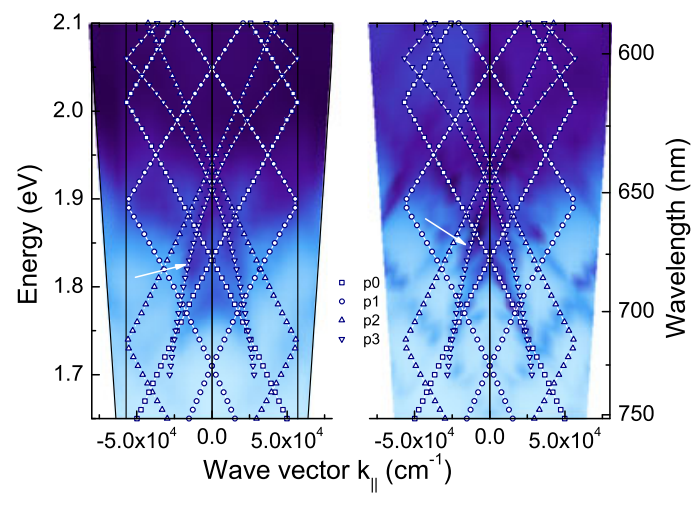

Fig. 11. Dispersion diagram of experimental (left) and modelled EQE (right) of the solar cell on the blazed grating under p-polarization. A weak asymmetry occurs to the left of the ordinate at $1.85 \mathrm{eV}$ (arrow). The superposed dispersion relations of an equivalent flat stack suggest that this is related to the crossing of zero and third order modes.

exceptions are the resonance at $675 \mathrm{~nm}$ and the weakly absorbing region beyond $700 \mathrm{~nm}$.

A presentation of the EQE data in the form of a dispersion diagram illustrates the mild asymmetry as seen in Fig. 11 for the case of p-polarization; again, s-polarization behaves similarly (not shown). On top of the color-coded EQE data, we superpose dispersion relations of the guided modes sustained in an equivalent stack of layers with flat interfaces which have been obtained by the pole method [16]. The most obvious asymmetry is observed for negative angles of incidence at about $1.85 \mathrm{eV}$, where modes of zero and third order cross. The asymmetric absorption enhancement for this condition is clearly visible in experimental and in theoretical data.

\section{SUMMARY}

We presented an adapted Rayleigh approach for the description of diffraction from purely sinusoidal and slightly anharmonic interface textures. These can appear in experimental situations where nonconformal growth tends to round out a given starting texture. We quantify our model against measured diffraction efficiencies from silver-covered gratings. Using a piecewise linear approximation of the shape, we find that the model yields numerically stable, but inaccurate results. Better agreement with the experiment is obtained when the shape of the interface is defined in terms of a sinusoidal with second harmonic, presumably because this type of trial function is continuous and differentiable. We applied our approach also to complete solar cells which requires the description of five media and four interfaces. Nonconformal coating by the deposition processes of the individual layers can be taken into account and noticeably improves the agreement of the modeling results with the experiment. The chosen approach may not be suitable for more complicated or even random surfaces, but it can help gain insight into absorption enhancement by the excitations of waveguide modes. Moreover, the model can relate the position and the strength of these resonances with the shape of the interface texture, which is represented by spatial Fourier series with a leading period and the amplitudes of the lowest order harmonic components. We think that this aspect makes our model helpful for designing an interface shape for a desired function, such as adapting the sensitivity to a certain wavelength or tailoring the average absorption enhancement over a range of wavelengths.

\section{REFERENCES}

[1] E. Yablonovitch and G. D. Cody, "Intensity enhancement in textured optical sheets for solar cells," IEEE Trans. Electron Devices, vol. ED-29, no. 2, pp. 300-305, Feb. 1982.

[2] P. Campbell and M. Green, "Light trapping properties of pyramidally textured surfaces," J. Appl. Phys., vol. 62, no. 1, pp. 243-249, 1987.

[3] D. Redfield, "Multiple pass thin film silicon solar cell," Appl. Phys. Lett., vol. 25, pp. 647-648, 1974.

[4] H. W. Deckman et al., "Optically enhanced amorphous silicon solar cells," Appl. Phys. Lett., vol. 42, no. 11, pp. 968-970, 1983.

[5] C. Haase and H. Stiebig, "Thin-film silicon solar cells with efficient periodic light trapping texture," Appl. Phys. Lett., vol. 91, no. 6, art. no. 0611162007.

[6] C. Rockstuhl et al., "Comparison and optimization of randomly textured surfaces in thin-film solar cells," Opt. Exp., vol. 18, no. 103, pp. A335-A341, 2010.

[7] V. E. Ferry, A. Polman, and H. A. Atwater, "Modeling light trapping in nanostructured solar cells," ACS Nano, vol. 5, no. 12, pp. 10055-10064, 2011.

[8] R. Dewan et al., "Optical enhancement and losses of pyramid textured thin-film silicon solar cells," J. Appl. Phys., vol. 110, no. 1, pp. 013101-1-013101-10, 2011.

[9] O. Isabella et al., "Full-wave optoelectrical modeling of optimized flattened light-scattering substrate for high efficiency thin-film silicon solar cells," Progr. Photovolt., Res. Appl., vol. 22, no. 6, pp. 671-689, 2012.

[10] M. Hammerschmidt et al. "Optical modelling of incoherent substrate light-trapping in silicon thin film multi-junction solar cells with finite elements and domain decomposition," Proc. SPIE, vol. 8980, pp. 898007-1-898007-8, 2014.

[11] J. W. Strutt, "On the dynamical theory of gratings," Proc. Roy. Soc. London, A, Containing Papers Math. Phys. Character, vol. 79, no. 532, pp. 399-416, 1907.

[12] R. Petit and M. Cadilhac, "Etude theorique de la diffraction par un reseau," CR Acad. Sci. Paris, vol. 259, pp. 2077-2080, 1964.

[13] P. M. Van Den Berg and J. T. Fokkema, "The Rayleigh hypothesis in the theory of reflection by a grating," J. Opt. Soc. Amer., vol. 69, no. 1, pp. 27-31, 1979

[14] S. H. Zaidi et al., "Characterization of thin Al films using grating coupling to surface plasma waves," J. Appl. Phys., vol. 71, no. 12, pp. 6039-6048, 1992.

[15] A. V. Tishchenko, "Numerical demonstration of the validity of the Rayleigh hypothesis," Opt. Exp., vol. 17, no. 19, pp. 17102-17117, 2009

[16] F.-J. Haug, K. Söderström, and C. Ballif, "Resonant absorption enhancement in solar cells with periodically textured interfaces," IEEE J. Photovolt., vol. 4, no. 3, pp. 785-790, May 2014.

[17] V. Kiselev, "Resonant conversion and reflection of surface waves in a thinfilm waveguide with a sinusoidally corrugated surface," Soviet J. Quantum Electron., vol. 4, pp. 182-184, 1974.

[18] F. Toigo et al., "Optical properties of rough surfaces: General theory and the small roughness limit," Phys. Rev., vol. B 15, no. 12, pp. 5618-5626, 1977.

[19] S. D. Gupta, G. Varada, and G. Agarwal, "Surface plasmons in two-sided corrugated thin films," Phys. Rev., vol. B 36, no. 12, pp. 6331-6335, 1987

[20] K. Söderström et al., "Photocurrent increase in n-i-p thin film silicon solar cells by guided mode excitation via grating coupler," Appl. Phys. Lett., vol. 96, pp. 213-508, 2010.

[21] P. B. Johnson and R. W. Christy, "Optical constants of the noble metals," Phys. Rev., vol. B 6, no. 12, pp. 4370-4379, 1972.

[22] F. J. Haug, A. Naqavi, and C. Ballif, "Diffraction and absorption enhancement from textured back reflectors of thin film solar cells," J. Appl. Phys., vol. 112, art. no. 024516, 2012.

[23] H. Raether, Surface Plasmons. Springer Tracts on Modern Physics. Berlin, Germany: Springer-Verlag, 1988, vol. 111.

Authors' photographs and biographies not available at the time of publication. 